are often significantly debilitated when they develop co-existent Aspergillus lung disease. They can present with worsening breathlessness, weight loss and significant haemoptysis. There is no consensus on how to best treat patients diagnosed with Sarcoid and CPA.

Methods A retrospective review of patients diagnosed with Sarcoidosis and CPA was performed. Cases were identified from a database held of patients diagnosed with CPA group in our specialist clinic. Patient demographics, presentation, laboratory parameters, radiology results were recorded and data analysed.

Results 38 patients with sarcoidosis were diagnosed with CPA from 2009 - March 2015. 23/36 (63\%) were male. 14/36 (38\%) patients were affected by breathlessness with MRC dyspnoea score $\geq 3.15 / 36(41 \%)$ of patients were on maintenance Prednisolone between $5-10 \mathrm{mg} /$ day and 3 patients on other steroid sparing agents. 12/28 patients had obstructive spirometry and average predicted gas transfer factor at 49\% (range $20-75 \%$ ).

$33 / 36$ (91\%) had CPA defined by presence of serum precipitins and $50 \%$ of patients had aspergillomas detected in the cavities on radiology. $27 / 36(75 \%)$ of patients were on antifungal therapy. On regular follow-up, antifungal therapy was titrated based on serum azole levels and as tolerated.

4/36 (11\%) patients had been treated for proven TB and 2/36 were on empirical treatment until $\mathrm{TB}$ diagnosis was excluded when there was clinical and radiological deterioration.

Conclusions Although sarcoidosis has slight female predominance, in this cohort of patients with CPA and sarcoid 63\% were males. Many of the patients had significant lung disease with high MRC dyspnoea scores, and obstructive spriometry often with low gas transfers. The management of CPA in the setting of sarcoidosis is complex due to the risk of immunosuppression and interactions with antifungal therapy. Close working between specialties is important in optimising such patients, to ensure the best patient outcomes.

\section{P35 IDENTIFYING NOVEL PREDICTORS OF OUTCOME IN SARCOIDOSIS}

P Minnis, M Poland, G Nolan, SC Donnelly. Department of Respiratory Medicine, Trinity Centre for Health Sciences, Tallaght Hospital, Dublin, Ireland

\subsection{6/thoraxjnl-2015-207770.172}

Background and objectives Sarcoidosis is a multisystem disease of unknown aetiology characterised by a Th1 granulomatous immune response. Predicting need for treatment in sarcoidosis is based on well-recognised prognostic indicators. ${ }^{1}$ We aimed to interrogate a large cohort of sarcoidosis patients in order to identify novel predictors for treatment and disease progression defined physiologically and radiologically.

Methods Clinical data from 338 patients with sarcoidosis was recorded retrospectively including; age at diagnosis, gender, Scadding CXR stage at presentation, presence of Erythema Nodosum (EN), baseline inflammatory markers, immunoglobulin levels, skin prick testing (SPT), pulmonary function tests, BAL results and vitamin D levels. There was a minimum follow up of 2 years for all patients. The primary outcome measure was the need for treatment, defined as requiring oral corticosteroids for 6 months or more. Secondary outcome measures included need for steroid sparing agents and progression defined radiologically using scadding criteria or HRCT when available and assessing pulmonary function test decline.
Results Multivariate analysis and modelling of all presenting features was performed. Patients were characterised into four groups-limited no treatment, limited requiring steroids, chronic but stable and chronic progressive steroid dependent. Overall the treatment rate was $50.4 \%$ in this cohort. In the chronic cohort 32\% required steroid sparing agents. Initial analysis confirmed previously known prognostic indicators including Scadding CXR stage, age $>40$ and presence of EN OR 0.24 (0.13$0.45), \mathrm{p}<0.0001$ at diagnosis. Additional predictors of prognosis included IgG levels, positive skin prick testing and vitamin D levels. In the group with elevated levels of IgG (IQR 15.7-19.8) at assessment the treatment rate was $77.1 \%$ OR 3.3 (1.5 to 7.6) $\mathrm{p}$ value 0.004 . In the group with positive SPT, only $17.4 \%$ required treatment, OR $0.21(0.07-0.62)$, p value 0.005 .

Conclusions These results indicate possible new markers of prognosis in terms of treatment and disease progression in sarcoidosis. Phenotyping sarcoid patients in this way may be valuable in designing future clinical trials in sarcoidosis.

\section{REFERENCE}

1 Neville E, Walker AN, Geraint James D. Prognostic factors predicting the outcome of sarcoidosis: an analysis of 818 patients. QJM 1983;4:525-533

\section{P36 ETHNIC DIFFERENCES IN COMPOSITE PHYSIOLOGIC INDEX (CPI) IN PULMONARY SARCOIDOSIS: A 10-YEAR EXPERIENCE IN A SPECIALIST SARCOIDOSIS CLINIC}

${ }^{1} \mathrm{~T}$ Tully, ${ }^{1} \mathrm{~J}$ Galloway, ${ }^{2} \mathrm{~J}$ Lally, ${ }^{1} \mathrm{E}$ Silber, ${ }^{1} \mathrm{P}$ Brex, ${ }^{1} \mathrm{~S}$ Walsh, ${ }^{1} \mathrm{G}$ Larkin, ${ }^{1} \mathrm{~J}$ Barker, ${ }^{1} \mathrm{~S}$ Birring. ${ }^{1}$ Kings College Hospital NHS Foundation Trust, London, UK; ${ }^{2}$ Kings College London, London, UK

\subsection{6/thoraxjnl-2015-207770.173}

Introduction and objectives CPI has been reported to be an accurate predictor of mortality in sarcoidosis and IPF. A CPI score of $>40$ is associated with a 5 year survival probability of $50 \% .{ }^{1}$ There is a paucity of studies of CPI in patients of black ethnicity. We investigated CPI in a large cohort of patients attending a specialist sarcoidosis clinic serving a large African Caribbean community in South-East London.

Methods 503 subjects with a diagnosis of sarcoidosis were assessed between May 2005 and May 2015. Relevant data needed to calculate CPI and ethnicity information was available in 297 patients with pulmonary involvement (based on radiological evidence $+/$ - histological evidence). We calculated CPI with the following formula: $91.0-(0.65 \times$ percent predicted DLCO $)$ - $(0.53 \times$ percent predicted FVC $)+(0.34 \times$ percent predicted FEV1). Clinical demographics, organ involvement, lung function, histology and subsequent therapy were systematically recorded (Table 1).

Results There were significant differences in CPI between all ethnic groups (ANOVA, p $<0.001$ ). Black patients had a higher mean CPI score compared to white patients (39.0 (14.2) vs 29.9 (18.5), $\mathrm{t}=-4.129, \mathrm{p}<0.001$ ) (Table 1 ). CPI was significantly higher in black Caribbean patients versus black African patients (41.2 (14.4) vs 36.3 (13.7), $\mathrm{t}=2.656 \mathrm{p}=0.008)$. CPI was weakly correlated with number of immunosuppressant medications used; correlation coefficient $\mathrm{r}=0.214, \mathrm{p}<0.001$. CPI was not associated with age at presentation, gender, initial requirement of treatment or prediction of $\geq 2$-organ involvement.

Conclusion Black patients have more severe disease than other ethnicities, with the most severe illness in those of black Caribbean ethnicity, who have the worst CPI scores. Further studies 
Poster sessions

Abstract P36 Table 1 Clinical characteristics of patients with pulmonary sarcoidosis at presentation

\begin{tabular}{|c|c|c|c|c|c|}
\hline & Black Caribbean $(n=108)$ & Black African $(n=105)$ & Other $(n=30)$ & White $(n=54)$ & Total population $(n=503)$ \\
\hline Age (mean \pm SD) & $42.1 \pm 12.0$ & $40.7 \pm 10.5$ & $44.8 \pm 10.0$ & $41.4 \pm 12.6$ & $41.7 \pm 11.2$ \\
\hline Female (\%) & 69.4 & 62 & 43.3 & 41 & 58.3 \\
\hline Organ involvement $>2$ ( $\%$ patients) & 70 & 63 & 70 & 59.3 & 63.1 \\
\hline Initial treatment with corticosteroids (\% patients) & 79.6 & 69.5 & 66.7 & 66.7 & 71.2 \\
\hline$\geq 2$ Immunosuppressant drugs required (\% patients) & 36 & 39 & 33 & 26 & 36.0 \\
\hline Elevated serum ACE (\% of group) & 79 & 84.2 & 65.2 & 58.1 & 71.7 \\
\hline FVC $(\%$ predicted \pm SD $)$ & $77.8 \pm 17.9^{*}$ & $80.2 \pm 15.8^{*}$ & $84.7 \pm 16.1$ & $93.2 \pm 18.2$ & $82.5 \pm 17.9$ \\
\hline $\mathrm{CPI}($ mean $\pm \mathrm{SD})$ & $41.8 \pm 14.3^{*}$ & $36.5 \pm 13.7^{*}$ & $32.8 \pm 15.2$ & $30.2 \pm 18.8$ & $36.9 \pm 15.6$ \\
\hline
\end{tabular}

${ }^{*} \mathrm{p}=<0.001$.

are needed to determine the long-term outcome in this patient cohort, such as decline in PFTs, quality of life and mortality.

\section{REFERENCE}

1 Walsh SL, Wells AU, Sverzellati N, et al. An integrated clinicoradiological staging system for pulmonary sarcoidosis: a case-cohort study. Lancet Respir Med. 2014;2:123-130

\section{Acute exacerbations of COPD and acute NIV}

\section{P37 FRAILTY AND ITS RELATIONSHIP TO MORTALITY IN PATIENTS RECEIVING ACUTE NON-INVASIVE VENTILATION (NIV) FOR RESPIRATORY FAILURE IN A DISTRICT GENERAL HOSPITAL}

${ }^{1} \mathrm{M}$ Hatton, 'D Daley, 'L Hughes, ${ }^{2} \mathrm{BC}$ Creagh-Brown, ${ }^{3} \mathrm{BC}$ Creagh-Brown. 'Respiratory Physiotherapy, Royal Surrey County Hospital NHS Foundation Trust, Guildford, UKi ${ }^{2}$ Respiratory and Intensive Care Medicine, Royal Surrey County Hospital NHS Foundation Trust, Guildford, UK; ${ }^{3}$ Surrey Peri-Operative Anaesthesia and Critical Care Collaborative Research Group (SPACeR), Faculty of Health and Medical Sciences, University of Surrey, Guildford, UK

\subsection{6/thoraxjnl-2015-207770.174}

Introduction NIV is a treatment for acute respiratory acidosis in patients admitted with exacerbations of COPD. BTS audits have demonstrated that NIV is frequently used for other conditions, functional dependency is common and in-hospital mortality is high. Tools to prognosticate outcome from acute use of NIV tend to be disease-specific. Targeting the use of NIV to those who are most likely to benefit from it should be a clinical and ethical priority. Frailty relates to patient outcome in clinical settings including elderly patients with COPD and critical care admissions. We hypothesised that patients with higher frailty scores who receive NIV for acute respiratory failure have inferior outcomes, compared to those assessed as being less frail.

Methods Prospective study of patients receiving acute NIV for respiratory failure; over 15 months. The respiratory physiotherapists manage all such patients and collect data into the EPR. An additional item of data was collected - the Clinical Frailty Scale (CFS). All analyses were by episode apart from demographics and mortality which were by individual.

Results 89 patients received 110 episodes of acute NIV, median age 79, (range 23-97) years. Diagnoses: COPD (56\%), Pneumonia and 'other' (30\%), cardiogenic pulmonary oedema (4\%), obesity-related (4\%), chest wall/neuromuscular (7\%). At initiation of NIV: median pH 7.26, $\mathrm{PaCO} 29.8 \mathrm{kPa}, \mathrm{PaO} 28.5 \mathrm{kPa}$.

Duration of hospital stay, median 16.5 days, in-hospital mortality (28\%). CFS median score 6 , range $(2-8)$. There was no correlation between CFS and age. CFS was statistically significantly higher in those who died (either during their index admission or during follow-up) than in those who survived - see Figure 1. Patients with a CFS of 7 or 8 had an in-hospital mortality of $40 \%$ and a total mortality of $80 \%$. By contrast those with a CFS of less than 7 had an in-hospital mortality of $24 \%$ and a total mortality of $46 \%$.

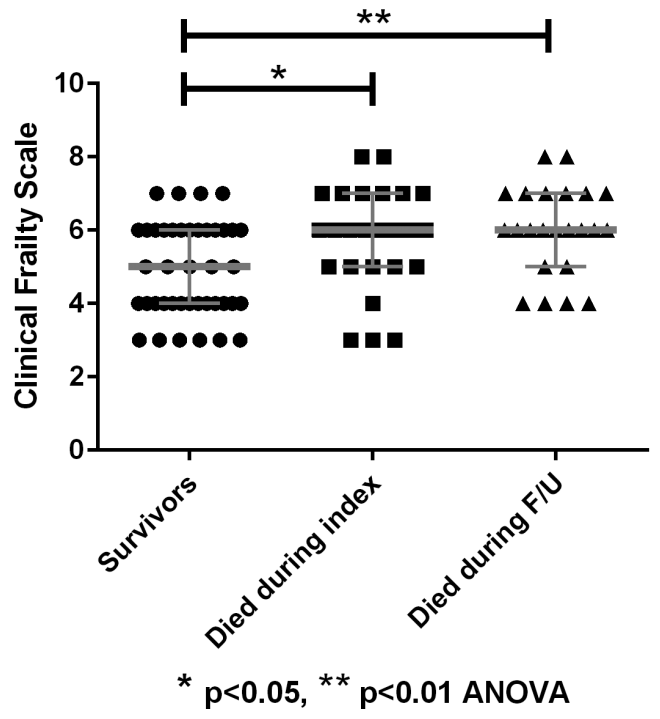

Abstract P37 Figure 1 Clinical Frailty Scale and outcome from acute NIV Median and IQR shown

Conclusions Patients receiving acute NIV who are very frail (CFS 7 or 8 ) are less likely to experience a mortality benefit. These data should inform discussions and decision-making about use of NIV.

\section{P38 IMPROVED MORTALITY AND OUTCOMES FOR PATIENTS REQUIRING NON-INVASIVE VENTILATION MANAGED IN A DEDICATED HYPER ACUTE MEDICAL UNIT}

T Buttle, H Chreif, H Woods, S Lohani. Darent Valley Hospital, Dartford, UK

\subsection{6/thoraxjnl-2015-207770.175}

Introduction Non-invasive ventilation (NIV) improves survival and outcomes in hypercapnic (type 2) respiratory failure. Following below average performance in a district hospital in the BTS national NIV audit, NIV delivery moved from acute medical and respiratory wards to a new hyper-acute medical unit (HAMU) providing level 1 nursing care and NIV. The unit is supervised by Respiratory Physicians and a dedicated NIV- 